\title{
Tuning Space Mapping: A Novel Technique for Engineering Design Optimization
}

\author{
Jie Meng, Slawomir Koziel, Senior Member, IEEE, John W. Bandler, Life Fellow, IEEE, \\ Mohamed H. Bakr, Member, IEEE, Qingsha S. Cheng, Member, IEEE
}

\begin{abstract}
School of Science and Engineering, Reykjavik University, Kringlunni 1, IS-103 Reykjavik, Iceland Department of Electrical and Computer Eng., McMaster University, Hamilton, ON, Canada L8S 4K1
\end{abstract}

\begin{abstract}
We introduce a tuning space mapping (TSM) technology for microwave design optimization. For the first time, we formulate the novel TSM concept and show how it relates to the standard space mapping methodology. The new method is based on a so-called tuning model that is created using engineering expertise and knowledge of the design problem, but also utilizes the efficiency of space mapping for translating the adjustment of the tuning parameters into relevant updates of the design variables. We illustrate our approach through optimization of a high-temperature superconducting (HTS) filter.

Index Terms-Computer-aided design (CAD), engineering optimization, space-mapping (SM), surrogate models, tuning.
\end{abstract}

\section{INTRODUCTION}

Space mapping (SM) is a widely recognized technique for optimization of expensive functions, also called "fine” models (typically implemented with CPU-intensive EM simulators), through iterative optimization and updating of the surrogate models which are built using cheaper "coarse" models [1]-[3]. SM has proved its success in many areas (e.g., [1]-[6]).

The concept of tuning, also widely used in microwave engineering [7], [8], can be considered within the scope of the SM approach. In our new tuning space mapping (TSM) algorithm, the surrogate model's role is taken by a so-called tuning model, which is constructed by introducing circuit-theory based components (e.g., capacitors, inductors or coupled-line models) into the fine model structure, and parameters of these circuit components are chosen to be tunable. In each iteration, the tuning model is updated and optimized with respect to the tuning parameters. This process takes little CPU effort as the

This work was supported in part by the Natural Sciences and Engineering Research Council of Canada under Grants RGPIN7239-06, RGPIN24978006, and STGP336760-06, and by Bandler Corporation.

J. Meng and Q.S. Cheng are with the Simulation Optimization Systems Research Laboratory, Department of Electrical and Computer Engineering, McMaster University, Hamilton, ON, Canada L8S 4K1.

S. Koziel was with the Simulation Optimization Systems Research Laboratory, Department of Electrical and Computer Engineering, McMaster University, Hamilton, ON, Canada L8S 4K1. He is now with the School of Science and Engineering, Reykjavik University, Kringlunni 1, IS-103 Reykjavik, Iceland.

J.W. Bandler is with the Simulation Optimization Systems Research Laboratory, Department of Electrical and Computer Engineering, McMaster University, Hamilton, ON, Canada L8S 4K1, and also with Bandler Corporation, Dundas, ON, Canada L9H 5E7.

M.H. Bakr is with the Computational Electromagnetics Research Laboratory, Department of Electrical and Computer Engineering, McMaster University, Hamilton, ON, Canada L8S 4K1. tuning model is typically implemented within a circuit simulator. With the optimal tuning parameters thus obtained, a calibration is needed to transform these tuning values into an appropriate modification of the design variables, which are then assigned to the fine model. The calibration process involves an auxiliary model, typically a fast space mapping surrogate. The TSM iteration is repeated until the fine model response is sufficiently close to the design target. The structure of the tuning model as well as a proper selection of tuning elements are crucial for the performance of the overall optimization process and normally require significant engineering expertise.

Approaches based on the idea of TSM have been proposed and applied in the microwave and RF arena although they are not explicitly formulated in the SM nomenclature. Rautio [7] introduced the tuning method developed by EPCOS for LTCC design and stated that it is an effective technique that can be used for any RF design. Swanson [8] successfully applied the port tuning strategy to microwave circuits such as combline filters.

In this paper, for the first time, we give a formal description of our novel tuning space mapping (TSM) optimization algorithm that iteratively updates and optimizes a so-called tuning model in conjunction with a calibration procedure which translates the adjustment of the tuning parameters into relevant adjustments of the design variable values. We illustrate the TSM algorithm using a simple microstrip line example and then use it to optimize a high-temperature superconducting (HTS) filter.

\section{TUNING SPACE MAPPING AlgORITHM}

We are concerned with the following optimization problem

$$
\boldsymbol{x}_{f}^{*}=\arg \min _{\boldsymbol{x}} U\left(\boldsymbol{R}_{f}(\boldsymbol{x})\right)
$$

where $\boldsymbol{R}_{f} \in R^{m}$ denotes the response vector of a fine model of the device of interest, $U$ is a merit function (e.g., minimax function or a norm), $\boldsymbol{x}$ is a vector of design parameters, and $\boldsymbol{x}_{f}{ }^{*}$ is the optimal solution to be determined.

The tuning space mapping (TSM) approach is an iterative optimization procedure that assumes the existence of two surrogate models: both are less accurate but computationally much cheaper than the fine model. The first model is a socalled tuning model $\boldsymbol{R}_{t}$ that contains relevant fine model data (typically a fine model response) at the current iteration point and tuning parameters (typically implemented through circuit elements inserted into tuning ports). The tunable parameters are adjusted so that the model $\boldsymbol{R}_{t}$ satisfies the design 
specifications. The conceptual illustration of the tuning model is shown in Fig. 1. The second model, $\boldsymbol{R}_{c}$ is used for calibration purposes: it allows us to translate the change of the tuning parameters into relevant changes of the actual design variables; $\boldsymbol{R}_{c}$ is dependent on three sets of variables: design parameters, tuning parameters (which are actually the same parameters as the ones used in $\boldsymbol{R}_{t}$ ), and space mapping parameters that are adjusted using the usual parameter extraction process [2] in order to have the model $\boldsymbol{R}_{c}$ meet certain matching conditions. Typically, the model $\boldsymbol{R}_{c}$ is a standard space mapping surrogate (i.e., a coarse model composed with suitable transformations) enhanced by the same or corresponding tuning elements as the model $\boldsymbol{R}_{t}$.

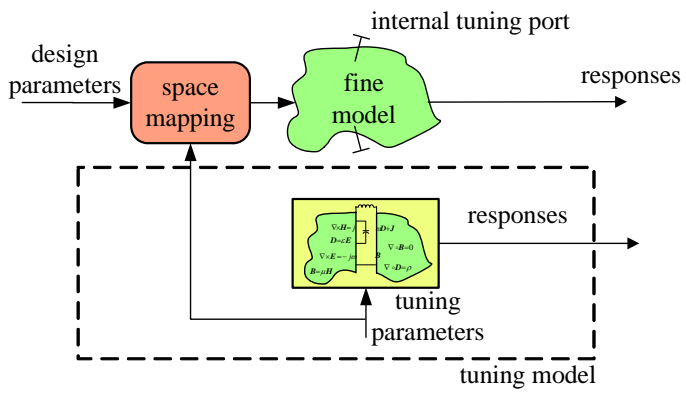

Fig. 1. The concept of the tuning model.

The TSM algorithm produces a sequence of points (design variable vectors) $\boldsymbol{x}^{(i)}, i=0,1, \ldots$. The iteration of the algorithm consists of two steps: optimization of the tuning model and a calibration procedure. First, the current tuning model $\boldsymbol{R}_{t}^{(i)}$ is built using fine model data at point $\boldsymbol{x}^{(i)}$. In general, because the fine model has undergone a disturbance, the tuning model response may not agree with the response of the fine model at $\boldsymbol{x}^{(i)}$ even if the values of the tuning parameters $\boldsymbol{x}_{\boldsymbol{t}}$ are zero, so that these values must be adjusted to, say, $\boldsymbol{x}_{t .0}{ }^{(i)}$ in order to obtain alignment:

$$
\boldsymbol{x}_{t .0}^{(i)}=\arg \min _{\boldsymbol{x}_{t}}\left\|\boldsymbol{R}_{f}\left(\boldsymbol{x}^{(i)}\right)-\boldsymbol{R}_{t}^{(i)}\left(\boldsymbol{x}_{t}\right)\right\|
$$

In the next step, we optimize $\boldsymbol{R}_{t}^{(i)}$ to have it meet the design specifications. We obtain the optimal values of the tuning parameters $\boldsymbol{x}_{t .1}{ }^{(i)}$ as follows:

$$
\boldsymbol{x}_{t .1}^{(i)}=\arg \min _{\boldsymbol{x}_{t}} U\left(\boldsymbol{R}_{t}^{(i)}\left(\boldsymbol{x}_{t}\right)\right)
$$

Having $\boldsymbol{x}_{t .1}{ }^{(i)}$ we perform the calibration procedure to determine changes in the design variables that yield the same change in the calibration model response as that caused by $\boldsymbol{x}_{t .1}{ }^{(i)}-\boldsymbol{x}_{t .0}{ }^{(i)}$. We first adjust the space mapping parameters $\boldsymbol{p}^{(i)}$ of the calibration model to obtain a match with the fine model response at $\boldsymbol{x}^{(i)}$

$$
\boldsymbol{p}^{(i)}=\arg \min _{\boldsymbol{p}}\left\|\boldsymbol{R}_{f}\left(\boldsymbol{x}^{(i)}\right)-\boldsymbol{R}_{c}\left(\boldsymbol{x}^{(i)}, \boldsymbol{p}, \boldsymbol{x}_{t .0}^{(i)}\right)\right\|
$$

The calibration model is then optimized with respect to the design variables in order to obtain the next iteration point $\boldsymbol{x}^{(i+1)}$

$$
\boldsymbol{x}^{(i+1)}=\arg \min _{\boldsymbol{x}}\left\|\boldsymbol{R}_{t}^{(i)}\left(\boldsymbol{x}_{t .1}^{(i)}\right)-\boldsymbol{R}_{c}\left(\boldsymbol{x}, \boldsymbol{p}^{(i)}, \boldsymbol{x}_{t .1}^{(i)}\right)\right\|
$$

Note that we use $x_{t .0}{ }^{(i)}$ in (4), which corresponds to the state of the tuning model after performing the alignment procedure
(2), and $x_{t .1}^{(i)}$ in (5), which corresponds to the optimized tuning model (cf. (3)). Thus, (4) and (5) allow us to find the change of design variable values $\boldsymbol{x}^{(i+1)}-\boldsymbol{x}^{(i)}$ necessary to compensate the effect of changing the tuning parameters from $\boldsymbol{x}_{t .0}{ }^{(i)}$ to $\boldsymbol{x}_{t .1}{ }^{(i)}$.

It should be noted that the TSM method exploits both the standard SM optimization, classical circuit and electromagnetic (EM) theory, as well as the engineer's expertise. For example, in a physics-based simulation according to classical EM theory, design parameters such as physical length and width of a microstrip line can be mapped to a "tuning component" such as an inductor or capacitor. The calibration process then transfers the tuning parameters to physical design parameters, which can be achieved by taking advantage of classical theory and engineering experience.

The TSM algorithm can be seen as a specialized case of a standard SM procedure that brings the concept of tuning into space mapping. In comparison with the standard SM [2], the TSM algorithm is equipped with an additional step: a calibration procedure that allows us to translate the adjustments of the tuning parameters into the relevant adjustments of the design variables. This step is necessary because there might be no straightforward relation between the tuning and design parameters in general.

\section{EXAMPLES}

\section{A. Microstrip Transmission Line}

In order to illustrate and clarify our TSM algorithm we use a simple example of a microstrip transmission line [7]. The fine model is implemented in Sonnet $\boldsymbol{e m}$ [9] (Fig. 2), and the fine model response is taken as the inductance of the line as a function of the line's length. The original length of the line is chosen to be $\boldsymbol{x}^{(0)}=400$ mils with width of 25 mils. A substrate with thickness $\mathrm{H}=25$ mil and $\varepsilon_{r}=9.8$ is used. Our goal is to find a length of line so that the corresponding inductance is $6.5 \mathrm{nH}$ at $300 \mathrm{MHz}$. The Sonnet $\boldsymbol{e m}$ simulation at $\boldsymbol{x}^{(0)}$ gives the value of $4.38 \mathrm{nH}$, i.e., $\boldsymbol{R}_{f}\left(\boldsymbol{x}^{(0)}\right)=4.38 \mathrm{nH}$.

We use the TSM algorithm of Section II. The tuning model $\boldsymbol{R}_{t}$ is developed by dividing the structure in Fig. 2 into two separate parts and adding the two tuning ports as shown in Fig. 3. A small inductor is then inserted between these ports as a tuning element. Note that the new version of Sonnet em allows the use of co-calibrated ports, which in this case has a negligible impact on the simulation results.

The tuning model is implemented in Agilent ADS [10] and shown in Fig. 4. The model contains the fine model data at the initial design in the form of the S4P element as well as the tuning element (inductor). Because of Sonnet's co-calibrated ports, there is perfect agreement between the fine and tuning model responses when the value of the tuning inductance is zero, so that $\boldsymbol{x}_{t .0}{ }^{(0)}$ is zero in our case.

Next, we optimize the tuning model to meet our target inductance $6.5 \mathrm{nH}$. The optimized value of the tuning inductance is $\boldsymbol{x}_{t .1}{ }^{(0)}=2.07 \mathrm{nH}$. 
Now, we need to perform the calibration step. We use the calibration model shown in Fig. 5 in which the dielectric constant of the microstrip element is used as a space mapping parameter $\boldsymbol{p}$. The value of this parameter is adjusted using (4) to 23.7 so that the response of the calibration model is $4.38 \mathrm{nH}$ at 400 mil, i.e., it agrees with the fine model response at $\boldsymbol{x}^{(0)}$.

The last step is to obtain the new value of the microstrip length, which is done according to (5). In particular, we optimize $\boldsymbol{x}$ (length of the line) with the tuning inductance set to $\boldsymbol{x}_{t .1}{ }^{(0)}=2.07 \mathrm{nH}$ to match the total inductance of the calibration model to the optimized tuning model response, $6.5 \mathrm{nH}$. The result is $\boldsymbol{x}^{(1)}=586 \mathrm{mil}$; the fine model response at $\boldsymbol{x}^{(1)}$ obtained by Sonnet $\boldsymbol{e m}$ simulation is $6.48 \mathrm{nH}$, which is acceptable.

This result can be further improved by performing a second iteration of the TSM algorithm, which gives the length of the microstrip line equal to $588 \mathrm{mil}$ and its corresponding inductance of exactly $6.5 \mathrm{nH}$.

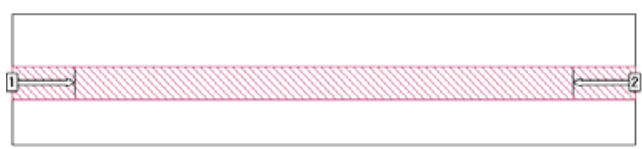

Fig. 2. The original structure of microstrip line in Sonnet.

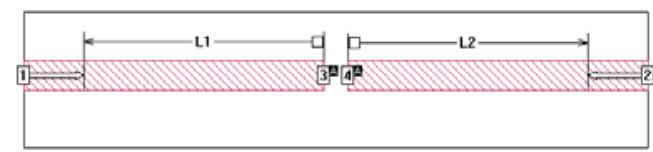

Fig. 3. The microstrip line under test after being divided and with inserted the co-calibrated ports.

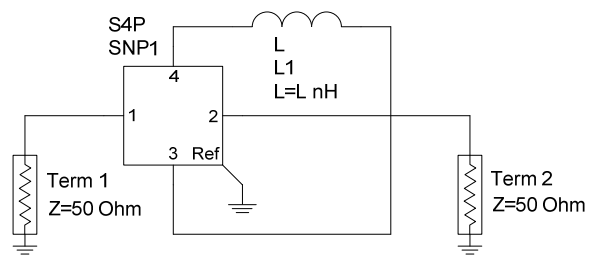

Fig. 4. Tuning model for the microstrip line design problem.

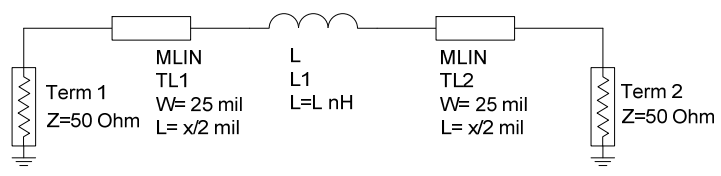

Fig. 5. Calibration model for the microstrip line design problem.

\section{B. High-Temperature Superconducting (HTS) Filter}

The high-temperature superconducting (HTS) bandpass filter [11] is shown in Fig. 6. The design parameters are the lengths of the microstrip coupled lines and the spacings between them: $L_{1}, L_{2}, L_{3}, S_{1}, S_{2}, S_{3}$. Design specifications are $\left|S_{21}\right| \geq 0.95$ for $4.008 \mathrm{GHz} \leq \omega \leq 4.058 \mathrm{GHz}$, and $\left|S_{21}\right| \leq 0.05$ for $\omega \leq 3.967 \mathrm{GHz}$ and $\omega \geq 4.099 \mathrm{GHz}$. The fine model is simulated in Sonnet $\boldsymbol{e m}$ [9] using a substrate of lanthanum aluminate with $\varepsilon_{r}=23.425$, height $H=20$ mil and loss tangent $=0.00003$. The width $W=7 \mathrm{mil}$ and the length of the input and output lines $L_{0}=50 \mathrm{mil}$.

The tuning model is constructed by dividing the five coupled line polygons in the middle and inserting the tuning ports at the new cut edges. Its S22P data file is then loaded into Agilent ADS. The circuit-theory coupled line components and capacitor components are chosen to be the tuning elements and are inserted into each pair of the tuning ports (Fig. 7). The lengths of the imposed coupled lines and the capacitances of the capacitors are assigned to be the tuning parameters, so that we have $\boldsymbol{x}_{t}=\left[\begin{array}{llll}L L_{1} & L L_{2} & L L_{3} & C_{1} C_{2} C_{3}\end{array}\right]^{T}\left(L L_{k}\right.$ in mils, $C_{k}$ in $\left.\mathrm{pF}\right)$.

The calibration model is implemented in ADS and shown in Fig. 8. It contains the same tuning elements as the tuning model. It basically mimics the division of the coupled lines performed while preparing $\boldsymbol{R}_{t}$. The calibration model also contains six (implicit) space mapping parameters that will be used as parameters $\boldsymbol{p}$ in the calibration process (4), (5). These parameters are $\boldsymbol{p}=\left[\begin{array}{llllll}H_{1} & H_{2} & H_{3} & \varepsilon_{r 1} & \varepsilon_{r 2} & \varepsilon_{r 3}\end{array}\right]^{T}$, where $H_{k}$ and $\varepsilon_{r k}$ are substrate height (in mils) and dielectric constant of the coupled line segment of length $L_{k}$ according to Fig. 6. Initial values of these parameters are $\left[\begin{array}{llllll}20 & 20 & 20 & 9.8 & 9.8 & 9.8\end{array}\right]^{T}$.

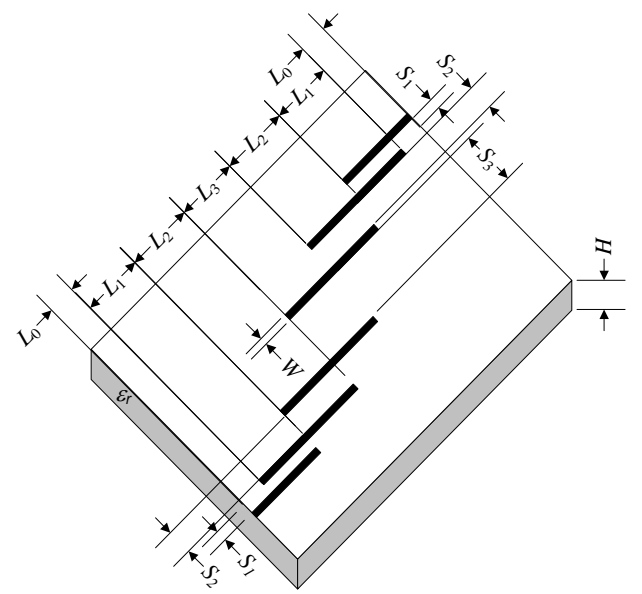

Fig. 6. HTS filter: Physical structure [11].

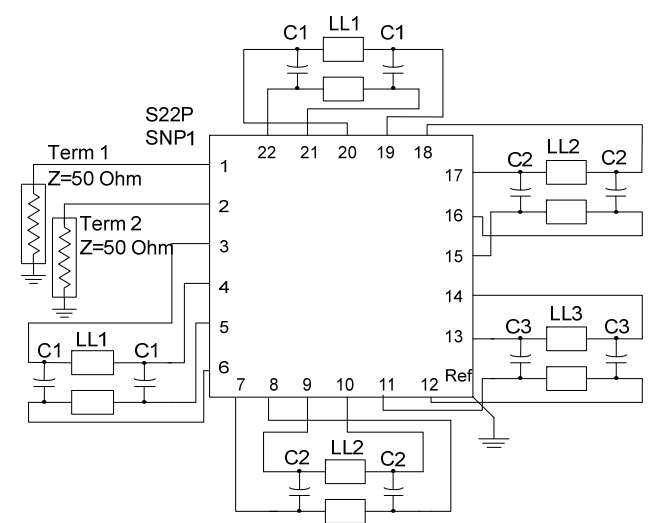

Fig. 7. HTS filter: tuning model (Agilent ADS).

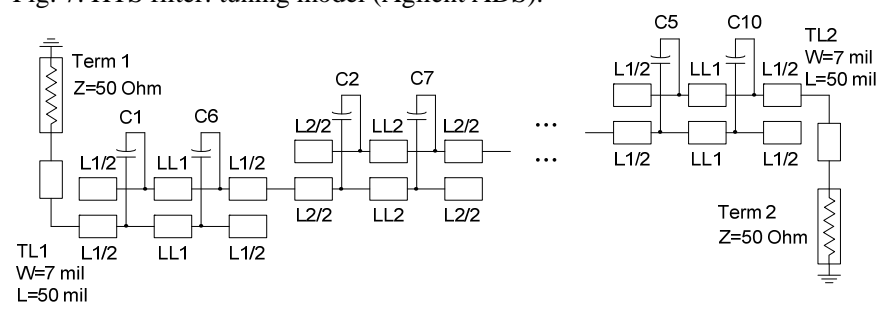

Fig. 8. HTS filter: calibration model (Agilent ADS). 


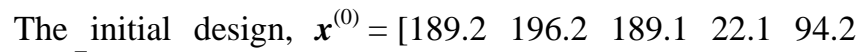
106.2 $]^{T}$ mil, is the optimal solution of the coarse model, i.e., the calibration model with zero values of the tuning parameters.

In this example, there is a small misalignment between the fine model (Sonnet $\boldsymbol{e m}$ ) response and the tuning model response with the tuning elements set to zero, even though the tuning elements have been inserted using the co-calibrated ports. Therefore, the alignment process (2) gives non-trivial

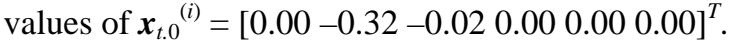

Fig. 9 shows the fine model response at the initial solution, and the response of the optimized tuning model. The tuning parameters obtained with (3) are $\boldsymbol{x}_{t .1}{ }^{(0)}=\left[\begin{array}{ll}3.06 & -3.07-3.04\end{array}\right.$ $0.00220 .00280 .0026]^{T}$. Note that some of the parameters take negative values, which is permitted in ADS.

Now, the calibration process must be performed in order to find the updated values of the design parameters. First, the space mapping parameters are adjusted using (4) to align the calibration model with the optimized tuning model for the values of tuning parameters equal $\boldsymbol{x}_{t .0}{ }^{(0)}$. We get $\boldsymbol{p}^{(0)}=[19.2$

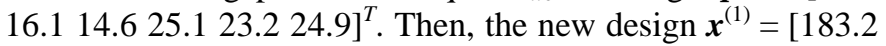
$195.7182 .621 .480 .985 .6]^{T}$ mil is found using (5). This solution already satisfies the design specifications, however, we perform the second TSM iteration to improve it further. The final design is obtained as $\boldsymbol{x}^{(2)}=\left[\begin{array}{ll}183.1 & 195.5183 .021 .0\end{array}\right.$ $83.787 .4]^{T}$ mil. Fig. 10 shows the fine model response at $\boldsymbol{x}^{(2)}$. The values of the design variables are summarized in Table I.

Note that the TSM algorithm requires only one iteration to satisfy the design specifications, and only one additional iteration to obtain an almost equal-ripple fine model response.

\section{CONCLUSION}

A tuning space mapping-an effective technique for microwave design is presented that brings together expertknowledge-based tuning and the efficiency of space mapping. We present the formal description of the tuning space mapping algorithm as well as provide examples that illustrate the concept and show its robustness. A highly optimized HTS filter design emerges after only three EM simulations.

\section{ACKNOWLEDGMENT}

The authors thank Dr. J.C. Rautio of Sonnet Software, Inc., for his helpful input on novel applications of his software.

\section{REFERENCES}

[1] J.W. Bandler, Q.S. Cheng, S.A. Dakroury, A.S. Mohamed, M.H. Bakr, K. Madsen, and J. Søndergaard, "Space mapping: the state of the art," IEEE Trans. Microwave Theory Tech., vol. 52, no. 1, pp. 337-361, Jan. 2004.

[2] S. Koziel, J.W. Bandler, and K. Madsen, "A space mapping framework for engineering optimization: theory and implementation," IEEE Trans. Microwave Theory Tech., vol. 54, no. 10, pp. 3721-3730, Oct. 2006.

[3] D. Echeverria and P.W. Hemker, "Space mapping and defect correction,” CMAM The Int. Math. J. Computational Methods in Applied Mathematics, vol. 5, pp. 107-136, 2005.

[4] M. Redhe and L. Nilsson, "Optimization of the new Saab 9-3 exposed to impact load using a space mapping technique," Structural and Multidisciplinary Optimization, vol. 27, pp. 411-420, 2004.
TABLE I

\begin{tabular}{cccc}
\multicolumn{4}{c}{ SONNET EM DESIGN PARAMETER VALUES OF THE HTS FILTER } \\
\hline \hline $\begin{array}{c}\text { Design } \\
\text { Parameters }\end{array}$ & Initial Solution & $\begin{array}{c}\text { Solution after } \\
\text { the First Iteration }\end{array}$ & $\begin{array}{c}\text { Solution after } \\
\text { the Second Iteration }\end{array}$ \\
\hline$L_{1}$ & $189.2 \mathrm{mil}$ & $183.2 \mathrm{mil}$ & $183.1 \mathrm{mil}$ \\
$L_{2}$ & $196.2 \mathrm{mil}$ & $195.7 \mathrm{mil}$ & $195.5 \mathrm{mil}$ \\
$L_{3}$ & $189.1 \mathrm{mil}$ & $182.6 \mathrm{mil}$ & $183.0 \mathrm{mil}$ \\
$S_{1}$ & $22.1 \mathrm{mil}$ & $21.4 \mathrm{mil}$ & $21.0 \mathrm{mil}$ \\
$S_{2}$ & $94.2 \mathrm{mil}$ & $80.9 \mathrm{mil}$ & $83.7 \mathrm{mil}$ \\
$S_{3}$ & $106.2 \mathrm{mil}$ & $85.6 \mathrm{mil}$ & $87.4 \mathrm{mil}$ \\
\hline \hline
\end{tabular}

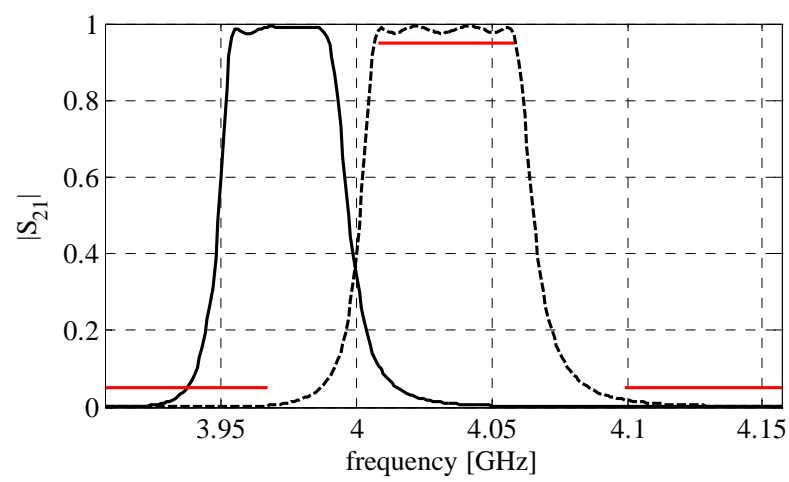

Fig. 9. HTS filter: fine model response at the initial design (solid line) and the response of the optimized tuning model (dashed line).

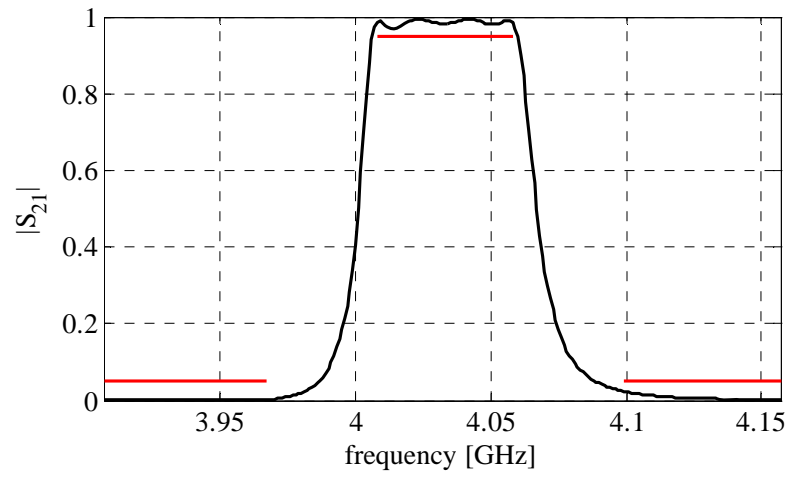

Fig. 10. HTS filter: fine model response $\left(\left|S_{21}\right|\right.$ obtained with Sonnet $\left.\boldsymbol{e m}\right)$ at the final design.

[5] G. Crevecoeur, L. Dupre, and R. Van de Walle, "Space mapping optimization of the magnetic circuit of electrical machines including local material degradation,” IEEE Trans. Magn., vol. 43, no 6, pp. 26092611, June 2007.

[6] M.F. Pantoja, P.Meincke, and A.R. Bretones, "A hybrid geneticalgorithm space-mapping tool for the optimization of antennas," IEEE Trans. Antenn. Propagat., vol 55, no. 3, pp. 777-781, March 2007.

[7] J.C. Rautio, "RF design closure-companion modeling and tuning methods," IEEE MTT IMS Workshop: Microwave component design using space mapping technology, San Francisco, CA, 2006.

[8] D.G. Swanson and R.J. Wenzel, "Fast analysis and optimization of combline filters using FEM," IEEE MTT-S Digest, Boston, MA, July 2001, pp. 1159-1162.

[9] $\boldsymbol{e m}^{\mathrm{TM}}$ Version 10.52, Sonnet Software, Inc., 100 Elwood Davis Road, North Syracuse, NY 13212, USA.

[10] Agilent ADS, Version 2003C, Agilent Technologies, 1400 Fountaingrove Parkway, Santa Rosa, CA 95403-1799, 2003.

[11] J.W. Bandler, R.M. Biernacki, S.H. Chen, R.H. Hemmers, and K. Madsen, "Electromagnetic optimization exploiting aggressive space mapping," IEEE Trans. Microwave Theory Tech., vol. 43, pp. 28742882, Dec. 1995 